\title{
LA BIOÉTICA COMO QUEHACER FILOSÓFICO
}

\author{
Jorge José Ferrer*
}

Resumen: El artículo examina el estatuto epistemológico de la bioética como disciplina académica. El autor sostiene que el estatuto epistemológico de un discurso lo determina la pregunta fundamental que se plantea y la respuesta que se busca, focos integradores del discurso. En el caso de la bioética, la pregunta fundamental es de índole moral. La bioética es pues una disciplina ética que tiene su hogar epistemológico en la filosofía. El autor también defiende el concepto de "éticas aplicadas". Sugiere finalmente que el método de la bioética, sobre todo la que se hace desde nuestras latitudes, debería adoptar el círculo hermenéutico como metodología para su filosofar.

Palabras clave: bioética, ética aplicada, epistemología, estatuto epistemológico, transdisciplinariedad, círculo hermenéutico

\section{BIOETHICS AS A PHILOSOPHICAL TASK}

Abstract: This article examines the epistemological statute of bioethics as an academic discipline. The author claims that the epistemological statute of a dialogue determines the fundamental question that is raised and the answer that is sought, essential points of discourse. In the case of bioethics, the fundamental question is of a moral nature. Therefore, bioethics is an ethical discipline that has its epistemological roots in philosophy. The author also defends the concept of applied ethics. Finally, he suggests that bioethical methods, above all especially what is realized in our latitudes, should adopt the Hermeneutic Circle as the methodology for its philosophizing.

Key words: bioethics, applied ethics, epistemology, epistemological statute, hermeneutic circle

\section{A BIOÉTICA COMO UM QUEFAZER FILOSÓFICO}

Resumo: $\mathrm{O}$ artigo examina o estatuto espistemológico da bioética como disciplina acadëmica. $\mathrm{O}$ autor defende que o estatuto espistemológico de um discurso é determinado pela pergunta fundamental que se faz e a resposta que se busca, focos integradores do discurso. No caso da bioética, a pergunta fundamental é de índole moral. A bioética é uma disciplina ética que encontra seu lugar epistemológico na filosofia. O autor também defende o conceito de "éticas aplicadas". O autor sugere finalmente que o método da bioética, sobretudo a que se faz a partir de nossas terras, deveria adotar o círculo hermenëutico como metodologia para seu filosofar.

Palavras chave: bioética, ética aplicada, epistemologia, estatuto epistemológico, transdisciplinariedade, círculo heremenêutico

\footnotetext{
* Doctor en Teología Moral. Catedrático del Departamento de Humanidades, Universidad de Puerto Rico en Mayagüez, Puerto Rico
} Correspondencia: jferrer@uprm.edu 


\section{¿Qué es la bioética?}

En la introducción a la segunda edición de su libro "The Foundations of Bioethics", publicado hace ya más de una década, H. Tristam Engelhardt señala que "bioética" es un sustantivo plural(1). En castellano diríamos que más que de "bioética" es preciso hablar de "bioéticas". En 2003, Juan Carlos Álvarez y el autor de este artículo publicamos un libro en el que se presentan críticamente algunos de los paradigmas teóricos más influyentes en las bioéticas contemporáneas(2,3). Como señala Marciano Vidal(4), además de los paradigmas basados en diversas orientaciones teóricas, se puede constatar también una correlación entre áreas geográfico-culturales y la diversidad de planteamientos bioéticos: "Así se habla de la bioética "europea" continental frente a la anglosajona y de bioética "mediterránea” frente a la bioética del norte (...) Son de destacar las peculiaridades que aportan las reflexiones bioéticas que se hacen desde el Tercer Mundo y, más concretamente, desde América Latina”.

No sólo son múltiples las orientaciones teóricas y los planteamientos culturales, sino que también podemos constatar distintos ámbitos de interés y de especialización dentro del mundo de la bioética. Así, por ejemplo, podemos hablar de bioética clínica, de la investigación científica, fundamental, bioética, etc.(5). Ante un mundo tan plural de orientaciones, tendencias y temas es imperioso preguntarse si hay algún denominador común que abarque todas las iniciativas y esfuerzos que se identifican con el sustantivo "bioética" y con los adjetivos que del mismo se derivan.

Opinamos que se debe dar una respuesta afirmativa a la pregunta precedente. Es preciso demarcar con claridad su identidad y su campo de acción propio: ¿Qué es la bioética? ¿Cuál es el objeto formal de su quehacer? ¿Cuáles los métodos justificados para llevar a cabo su tarea disciplinaria? No es insólito escuchar planteamientos que parecerían insinuar una especie de imperialismo o totalitarismo que subsumiría todos los problemas morales bajo la égida de la bioética. Consideramos que tal pretensión no es sólo inexacta. Va en detrimento de la propia bioética: si todo es bioética, ¿en qué consiste su peculiar aportación al concierto de las ciencias y las disciplinas académicas?

En la introducción a la segunda edición de la Encyclopedia of Bioethics, Warren T. Reich, su editor principal, presenta una definición de bioética que consideramos sigue siendo válida: "El estudio sistemático de las dimensiones morales -incluyendo la visión moral, las decisiones, las conductas y las políticas- de las ciencias de la vida y del cuidado de la salud, adoptando diversas metodologías éticas y en un contexto interdisciplina$\operatorname{rio}(6)$.

Tendríamos que decir que la bioética es, ante todo, un discurso ético, que se distingue de otros discursos éticos por el objeto material del que se ocupa: los problemas morales que se plantean en esos campos de actividad humana que son las ciencias de la vida y las profesiones sanitarias. Por eso coincidimos con las consecuencias que Marciano Vidal, el ya citado teólogo moralista, deriva de la definición de Reich: "...la bioética es formalmente una rama o subdisciplina del saber ético, del que recibe su estatuto epistemológico básico y con el que mantiene una relación de dependencia justificadora y orientadora" (4:186). Sostenemos pues que, epistemológicamente hablando, la bioética es una de las llamadas éticas aplicadas, y éstas, a pesar del análisis interdisciplinario que requieren los problemas que abordan, constituyen en definitiva empresas filosóficas. Justificar estas afirmaciones es la tarea que nos proponemos completar en los párrafos que siguen.

\section{El hogar epistemológico de la bioética}

Uno de los problemas que debe enfrentar cualquiera disciplina es la determinación de su estatuto epistemológico. Entendemos por epistemología el estudio de la naturaleza del conocimiento y de su justificación(7). El filósofo mexicano León Olivé la define en término parecidos. Según este último autor, es “...la disciplina que analiza críticamente las prácticas cognitivas, es decir, aquellas mediante las cuales se genera, aplica y evalúan diferentes formas de conocimiento...”(8). La epistemología se pregunta cómo conocemos y cómo podemos fundamentar nuestros conocimientos. Cuando hablamos del estatuto epistemológico de una disciplina académica nos referimos a la justificación racional de sus métodos y sus saberes. Sin esa justificación, la disciplina no tiene un lugar en el conjunto de las disciplinas académicas que aspiran a generar un saber científico, en el sentido amplio del término. Entendemos aquí por "ciencia" un saber sistemático, metodológicamente riguroso y racionalmente justificado, dentro de los límites tolerados por su objeto de 
estudio $^{1}$. La identidad disciplinaria de un saber determinado depende precisamente de la definición de su identidad epistemológica. Señala el estudioso italiano Salvatore Privitera: "Indagar sobre la estructura lógica de una ciencia concreta (...) significa indagar sobre la condición indispensable de su existir en cuanto ciencia, y en cuanto ciencia distinta de las otras ciencias. Condición indispensable del existir de una ciencia es la especificidad de su estatuto epistémico, que constituye al mismo tiempo el principio de identidad y el de diversidad de esa misma ciencia concreta"(9).

La identidad epistemológica la determina la pregunta fundamental que guía la investigación en una determinada disciplina; aquello que los escolásticos llamaban su "objeto formal". En otra parte hemos escrito: "El estatuto epistemológico del conjunto del discurso lo determina la pregunta que se plantea y la respuesta última que se busca, la perspectiva dominante del análisis"(10). Si por ética entendemos la disciplina académica cuyo problema central consiste en "la fundamentación de normas legítimas de convivencia para la acción y la interacción entre seres humanos, aunque sus morales sean diversas" (8:135), no cabe duda de la identidad ética de la bioética². Es, como se ha dicho, la ética para las ciencias de la vida y las profesiones de la salud en una sociedad pluralista, es decir, con múltiples morales positivas en su seno. Esto nos lleva al problema de la identidad de las llamadas "éticas aplicadas" y si la bioética puede considerarse una de ellas.

\section{Las éticas aplicadas}

El primer número del Kennedy Institute of Ethics Journal de 2007 está dedicado al tema de la bioética como ética aplicada (Is Bioethics Applied Ethics?) El artículo principal lo firman Robert Baker y Laurence McCullough(11), autores que rechazan el modelo de las "éticas aplicadas" para definir la bioética. Dicho modelo exige, en su opinión, que el bioeticista aplique una teoría ética -el utilitarismo o el deontologismo

1 Es evidente que damos al término "ciencia" un sentido amplio, que va más allá de las ciencias empíricas. Lo usamos de manera intercambiable con los términos "disciplina académica" y "saberes académicos", aunque reconocemos que es un uso que seguramente no todos aceptarían.

2 Es una definición minimalista porque parecería reducir la ética a la sola ética normativa. Desde nuestro punto de vista, la ética abarca un campo mucho más amplio que incluye la justificación del deber moral y la elaboración de teorías éticas debidamente fundamentadas. Sin embargo, para los fines de este trabajo nos basta la definición minimalista de Olivé. kantiano, por ejemplo-a la resolución de los problemas concretos de las ciencias de la vida o en las profesiones de la salud. A partir de esta definición del modelo de las éticas aplicadas, Baker y McCullough sostienen que, históricamente, los autores que se han ocupado de los problemas relativos a la ética médica no han procedido de esa manera ${ }^{3}$. Aunque admiten que la filosofía moral ha tenido influencia en el desarrollo de la ética médica, el paradigma adoptado por los bioeticistas ha sido uno de "apropiación" y no de "aplicación". Por "apropiación" entienden la adopción de conceptos filosóficos, adaptándolos para usarlos para los propios fines en un determinado contexto práctico, desgajándolos así del marco teórico en el que han tenido su origen.

¿Nos obliga la investigación de estos autores a descartar el modelo de la ética aplicada para hablar de la identidad y del estatuto epistemológico de la bioética? Para comprender nuestra posición vamos a referirnos, en primer lugar, al artículo publicado en el mismo número del Kennedy Institute of Ethics Journal por Tom L. Beauchamp(12). Este último sostiene, con razón, que la definición de ética aplicada del artículo de Baker y McCullough es demasiado restrictiva. Parecería que ellos reducen las "éticas aplicadas" a la metodología habitualmente conocida como "deductivismo": los juicios morales se deducen directamente de preceptos normativos generales, pertenecientes a una determinada estructura teórica preexistente. Es decir, en una concepción estrictamente deductivista, todos los juicios morales fundamentados serían de índole silogística: la teoría brindaría los principios generales y a partir de estos se deducirían juicios fundamentados acerca de prácticas humanas determinadas.

Es verdad que los juicios silogísticos pueden jugar un papel válido en la valoración moral de las acciones particulares, especialmente en sistemas naturalistas deontológicos, pero eso no significa que los autores que entienden la bioética-o la ética empresarial, ingenieril, de la investigación o de la informática, por ejemplocomo ética aplicada reduzcan su metodología a la deducción de conclusiones morales a partir de un determinado marco teórico. Más aún, como señala Beauchamp, ninguno de los autores que escribe sobre estos temas lo hace. El citado autor nos ofrece una definición

3 Aunque la bioética no se identifica con la ética médica, el artículo de los autores que estamos citando se centra en el estudio de la ética médica, que mutatis mutandi podemos aplicar a otras éticas aplicadas, incluyendo la bioética. 
de las éticas aplicadas que encontramos enteramente aceptable: "Ética aplicada" se usa hoy para referirse, en términos amplios, a cualquier uso de los métodos de razonamiento para examinar críticamente decisiones morales prácticas en las profesiones, la tecnología, la política pública y otros campos similares. El término "aplicado" se refiere habitualmente al uso de métodos filosóficos -incluyendo análisis conceptuales, equilibrio reflexivo, casuística (...) y otros semejantes- para examinar críticamente problemas, prácticas y políticas que tienen una dimensión moral"(12:56).

Por lo tanto, concluye Beauchamp, el método de apropiación que proponen Baker y McCullough es una forma de ética aplicada, no su negación. Concurrimos con el juicio de Beauchamp: la bioética es una ética aplicada en cuanto adopta métodos de análisis filosófico -es decir, métodos estrictamente racionales de análisis moral para llegar a conclusiones racionalmente justificadas- con el fin de examinar problemas morales concretos que se plantean en las ciencias de la vida y en las profesiones de la salud. No estamos defendiendo primordialmente el término "ética aplicada", que quizá no sea el más feliz, si tenemos en cuenta los malos entendidos a los que parece dar lugar ${ }^{4}$. Nuestra defensa se centra en la identidad genuinamente ética y por ende filosófica del quehacer bioético.

En nuestro ámbito lingüístico, Adela Cortina se ha ocupado ampliamente del problema del estatuto epistemológico de las éticas aplicadas. También esta autora rechaza una simplista comprensión deductivista de las éticas aplicadas: "Porque la expresión "ética aplicada" (...) despierta de inmediato la impresión de que se trata de una disciplina que funciona de modo deductivo; es decir, de una disciplina que, una vez descubiertos los principios éticos, se limita a aplicarlos a los ámbitos y los casos concretos, siguiendo el modelo de racionalidad del silogismo deductivo"(13).

Cortina señala que esta comprensión no representa, en modo alguno, el proceder de las éticas aplicadas. Los problemas surgen en los distintos ámbitos de la vida -en nuestro caso, en la investigación científica biomédica y en la praxis clínica- y están dotados de peculiaridades específicas. Son estos problemas particulares los que constituyen el punto de partida de la reflexión en bio-

4 Halley Sánchez propone, por ejemplo, el de "éticas contextualizadas" (comunicación personal al autor de este artículo el 27 de junio de 2008). ética y en las restantes éticas aplicadas: “...la reflexión de la ética aplicada -a pesar del nombre que ostentafunciona más "de abajo arriba" que de arriba abajo, más desde la base republicana de las distintas esferas que desde la monarquía del saber filosófico"(13:165). La ética es "aplicada" si se esfuerza por dar respuestas fundamentadas a los problemas morales que se plantean en los campos siempre concretos del obrar humano, no en cuanto aplica unos principios eternos y predeterminados a unas disciplinas particulares. Sostenemos que la bioética es una ética aplicada en este sentido del término. Y en cuanto "ética" es filosofía. Pero también esta tesis encuentra reparos en el pensamiento de reconocidos autores.

\section{Interdisciplinariedad, transdisciplinariedad y bioética}

La identidad filosófica de la bioética se podría cuestionar a partir del abordaje interdisciplinario o transdisciplinario que suelen requerir los problemas de los que se ocupa esta reflexión. Esta es la opinión que sostiene, por ejemplo, el reconocido bioeticista colombiano Gilberto Cely, reflexionando desde una matriz potteriana: "Interpretando el pensamiento de Potter, nuestra propuesta implica una visión transdisciplinaria de la reflexión ética sobre los seres vivos, incluyendo al hombre (...) En esta comprensión transdisciplinaria y global, la bioética, en virtud de su inspiración ecológica, es una ciencia de interfases; por consiguiente, no es una simple ética aplicada y normativa, como piensan algunos filósofos, y tampoco es correcto desmembrarla en bioéticas sectoriales sin interacción alguna(14).

Probablemente la principal autoridad sobre transdisciplinariedad sea el físico rumano Basarab Nicolescu(15). Como bien señala su traductora al inglés, Karen-Claire Voss, la transdisciplinariedad es ante todo una actitud ante el mundo y ante la vida, que tiene en cuenta la enorme complejidad de la realidad y que rehúsa por tanto encerrarse en antiguos moldes disciplinarios que todavía compartimentan la búsqueda de la verdad en nuestras universidades. Según Nicolescu, la pluridisciplinariedad consiste en el estudio de un objeto por varias disciplinas a la vez. Por ejemplo, un cuadro de Giotto se puede estudiar desde la historia, la geometría y la química, llegando así a un conocimiento más cabal del objeto de estudio. La interdisciplinariedad alude a la transferencia de métodos de una ciencia a otra, dando origen a disciplinas noveles. Por ejemplo, los métodos 
de la física nuclear se pueden aplicar a la medicina clínica, dando lugar a nuevas terapias oncológicas y a todo el campo de la medicina nuclear ${ }^{5}$. Por último, la transdisciplinariedad busca conocer lo que transciende a todas las disciplinas, lo que está "simultáneamente (...) entre las disciplinas, a través de las diferentes disciplinas y más allá de toda disciplina ${ }^{\text {"“. }}$

La bioética sería transdisciplinaria porque aborda el complejísimo problema de la vida en este planeta (y quizás un día también sea preciso ocuparse de la vida allende nuestro planeta). Su quehacer exige que se incorporen los aportes de la biología y las demás ciencias de la vida. Pero también son indispensables las perspectivas de las ciencias sociales, el derecho, las filosofías tradicionales y hasta las diversas tradiciones religiosas y espirituales. Y de esa pluralidad de perspectivas nacen otras inéditas, impensables sin dicha sinergia transdisciplinaria, dando lugar a conocimientos que trascienden las posibilidades de los campos tradicionales. A partir de estos planteamientos, Cely concluye que la bioética "...no es una simple ética aplicada y normativa, como piensan algunos filósofos.” Pero para ese autor eso no significa que deje de ser genuina ética, una reflexión encaminada a favorecer "...que el ser humano construya un comportamiento coherente con la lógica de la vida, una ética que favorezca el cultivo virtuoso y feliz (agatístico) de la vida en todas sus manifestaciones"(14:73).

Desde nuestro punto de vista, el método de análisis de los problemas tratados en bioética exige un abordaje transdisciplinario o al menos interdisciplinario. Pero una vez hemos aceptado la transdisciplinariedad como actitud y como método de análisis no podemos negar que estamos haciendo ética, como acepta el propio Cely y, hasta donde hemos podido investigar, todos los autores que se ocupan de estos temas. Es decir, el foco integrador del discurso bioético es de índole moral. La pregunta de fondo en bioética y que confiere

5 Podría quizá cuestionarse si son los métodos o más bien los hallazgos de la física nuclear los que se aplican a la terapia oncológica, pero el punto es secundario para nuestros fines.

6 La lectura de estas explicaciones no puede dejar de suscitar la siguiente pregunta en el lector iniciado en la historia de la filosofía: ¿No es esto en definitiva lo que ha querido hacer, al menos en buena medida, la tradición filosófica occidental? Pensamos que la transdisciplinariedad misma es una actitud genuinamente filosófica. ¿¿No será, quizá, el discurso sobre la transdisciplinariedad un necesario "redescubrimiento del Mediterráneo", como respuesta al olvido de la vocación transdisciplinaria del quehacer filosófico por muchos que ejercen de filósofos en nuestras instituciones académicas? unidad e identidad epistemológica al discurso no se refiere sólo a cuestiones de hecho sino esencialmente a los juicios axiológicos justificados en la situación o situaciones bajo estudio, juicios que orientarán en la toma de decisiones en orden a una praxis que contribuya al genuino bien de las personas en la comunidad de personas; es decir, a la realización del bien moral, al cultivo "agatístico" de la vida del que nos habla el propio Cely.

Pero precisamente porque estamos haciendo ética cuando trabajamos en clave bioética se impone concluir que la bioética encuentra en la filosofía su hogar epistemológico, su identidad última, sin que esto signifique que se trate, como ya hemos explicado, de la simple aplicación mecánica de teorías y conceptos filosóficos tradicionales a situaciones particulares. Aun cuando el bioeticista encuentre inspiración y metodologías en las tradiciones filosóficas, se ve precisado a trabajarlas creativamente, para que devengan instrumentos aptos en la búsqueda de soluciones morales justificadas, en el contexto de las sociedades pluralistas y complejas que habitamos. Pero por pluralistas y complejas que pretendan ser estas sociedades, no pueden renunciar a ser justas y a crear espacios en los que sus ciudadanos puedan florecer en la vida buena, conforme a las diversas cosmovisiones felicitantes que sean compatibles con la justicia y el bien común.

\section{Filosofía y compromiso transformador: a modo de conclusión}

Sospechamos que la resistencia a ubicar la bioética en el hogar filosófico proviene de la identificación de la filosofía con una actividad distante del quehacer comprometido con la transformación de la realidad. Esta concepción de la filosofía tiene sus fundamentos en la esterilidad de algunos de los debates que no pocas veces se han planteado, y que quizá se siguen planteando, en los medios en los que se cultiva la filosofía académica. Sin embargo, nada está más lejos de su más genuina tradición. Una filosofía comprometida con los problemas reales del bien humano puede trazar su genealogía por lo menos hasta Sócrates. Dicho de otro modo, sospechamos que la resistencia a fijar el hogar epistemológico de la bioética en el país filosófico nace precisamente de una concepción del filosofar que responde todavía a los esquemas mentales y práxicos rechazados por Marx en la undécima de sus tesis sobre Feuerbach: "Los filósofos no han hecho más que inter- 
pretar el mundo, pero de ahora en adelante se trata de transformarlo"(16). El filosofar propio de la bioética no puede limitarse a interpretar el mundo. De poco o nada sirve si no contribuye a transformarlo. Debe ser una reflexión comprometida con cursos de acción que promuevan el bien integral de los humanos y de los demás vivientes en nuestro planeta.

La visión y análisis transdisciplinarios en el abordaje de los problemas no está en modo alguno reńido con la identidad filosófica de la bioética. Lo propio de la filosofía ha sido siempre esa visión amplia, que se pregunta por el sentido de la totalidad y por el significado último de las cosas. Una filosofía que se limitase a los debates entre los así llamados "filósofos profesionales" habría renunciado a su más genuina identidad. Si bien es útil que algunos se dediquen con especial atención al estudio de la historia de la filosofía y de los métodos de análisis tradicionalmente asociados con ella, no se puede olvidar que el filosofar es patrimonio común de la humanidad. Ninguna persona que sea capaz de pensar e interrogar puede sustraerse a las cuestiones filosóficas.

Los que hacemos bioética en las latitudes latinoamericanas no deberíamos ignorar las aportaciones hechas por los debates sobre el método de la teología y la filosofía de la liberación durante la segunda mitad del pasado siglo, particularmente en las décadas de los setenta y los ochenta. A modo de ejemplo, quisiera hacer referencia a la obra del desaparecido teólogo uruguayo Juan Luis Segundo (1925-1996), autor del libro "Liberación de la teología”. En dicha obra, Segundo propone la idea del método teológico como un círculo hermenéutico que parte de los hechos de la experiencia y lleva a una reflexión que conduce, a su vez, a una nueva praxis. Pero el círculo no termina: perpetuamente exige apertura a los nuevos cuestionamientos que incesantemente plantea la realidad.

No estamos haciendo aquí teología. Queremos hacer bioética con una metodología filosófica, es decir, estrictamente racional y por ende secular (y no secularista) ${ }^{8}$. Pero sugerimos que los planteamientos sobre el método teológico de Segundo nos pueden iluminar sobre la metodología que debe adoptar esa peculiar manera de hacer filosofía que es la bioética: los datos de la realidad, siempre cambiantes, nos obligan a formular continuamente preguntas inéditas que exigen una reflexión moral. Dicha reflexión debe contribuir a la generación de nuevas praxis, axiológicamente fundamentadas, que a su vez serán continuamente evaluadas y renovadas debido al imparable desarrollo de las ciencias y de la vida misma.

En resumen, la bioética es una praxis filosófica que busca la realización de los valores morales en las prácticas relacionadas con las ciencias vidas y las profesiones de la salud, dentro del marco de un compromiso existencial con la transformación de la realidad en orden al bien común, a la justicia y al florecimiento de la vida en este planeta azul. Su actitud y sus métodos de análisis son transdisciplinarios; su identidad y su hogar epistemológico último se encuentran en la filosofía.

7 Publicado en español en 1973 por la editorial Carlos Lohlé de Buenos Aires. La traducción inglesa, "The Liberation of Theology", fue publicada por Orbis Books en 1976.

8 Sin que ello signifique que no sea legítimo hacer una bioética teológica, anclada en una determinada tradición de fe. No puede olvidarse la importancia de la aportación de los teólogos católicos y protestantes -McCormick, Ramsey, Fletcher y tantos otros- en el nacimiento de la moderna bioética. Mucho menos queremos sugerir que para hacer bioética sea preciso adoptar una actitud secularista, que rechace todo lo religioso. Lamentablemente en algunos ambientes se constata esta errada actitud. De otra parte, también es preciso afirmar la autonomía de la razón bioética secular frente a indebidas tutelas autoritarias. 


\section{Referencias}

1. Engelhardt HT. The Foundations of Bioethics. New York: Oxford University Press; 1996: 3-31.

2. Ferrer JJ, Álvarez JC. Para fundamentar la bioética. Madrid y Bilbao: Universidad Pontificia Comillas y Desclée de Brouwer; 2005.

3. Ferrer JJ. Deber y deliberación. Mayagüez (Puerto Rico): Centro de Publicaciones Académicas de la Universidad de Puerto Rico en Mayagüez; 2007: 13-125.

4. Vidal M. Orientaciones éticas para tiempos inciertos. Bilbao: Desclée de Brouwer; 2007: 191-192.

5. Callahan D. Bioethics. En: Post SG, (ed.) Encyclopedia of Bioethics, t. 1: 281-282.

6. Reich WT. Introduction. En Reich WT, (ed.) Encyclopedia of Bioethics. New York: Simon and Schuster Macmillan; 1995, t. 1: xxi.

7. Noser PK. Epistemology. En: Audi R, (ed.) The Cambridge Dictionary of Philosophy. Cambridge: Cambridge University Press; 1995: 233.

8. Olivé L. Epistemología en la ética y en las éticas aplicadas. En: Garrafa V, Kottow M, Saada A. Estatuto epistemológico de la bioética. México: Universidad Nacional Autónoma de México y Red Latinoamericana y del Caribe de Bioética de la UNESCO; 2005: 136.

9. Privitera S. Epistemología moral. En: Compagnoni F, Piana G, Privitera S, Vidal M, (eds.) Nuevo diccionario de teología moral. Madrid: Paulinas; 1990: 551.

10. Ferrer JJ. Historia y fundamentos de los comités de ética. En Martínez JL, (ed.) Comités de bioética. Madrid y Bilbao: Universidad Pontificia Comillas y Desclée de Brouwer; 2003: 41.

11. Baker R, Mc Cullough L. Medical Ethics' Appropriation of Moral Philosophy: The Case of the Sympathetic and the Unsympathetic Physician: Kennedy Institute of Ethics Journal 2007; 17: 3-22.

12. Beauchamp TL. History and Theory in "Applied Ethics": Kennedy Institute of Ethics Journal 2007; 17: 55-64.

13. Cortina A. Ética aplicada y democracia radical. Madrid: Tecnos; 1997: 167.

14. Cely Galindo G. Bioética global. Bogotá: Editorial Pontificia Universidad Javeriana; 2007: 79.

15. Nicolescu B. Manifesto of Transdisciplinarity. New York: State University of New York Press; 2003.

16. Marx K. Tesis sobre Feuerbach. Sitio en Internet. Disponible en http://www.marxists.org/espanol/m-e/1840s/45-feuer. htm

Recibido: 9 de marzo de 2009

Aceptado: 15 de marzo de 2009 